EPJ Web of Conferences 32, 03010 (2012)

DOI: $10.1051 /$ epjconf/20123203010

(C) Owned by the authors, published by EDP Sciences, 2012

\title{
ECE COMPLEX ON T-10 TOKAMAK
}

\author{
V.I. Poznyak, V.V. Pitersky, G.N. Ploskirev, E.G. Ploskirev \\ RRC “Kurchatov Institute”, IFT, 123182, Kurchtov Sq. 1, Moscow, Russia, pozn@tokamak.ru
}

\begin{abstract}
The structure and main characteristics of ECE complex on T-10 are presented. T-10 ECE system possesses by certain features: measurements are fulfilled simultaneous on $1^{\text {st }}$ and $2^{\text {nd }}$ harmonics; frequency range of every harmonic is widened down; ECE measurements are made in two polarization at the same time; antenna system has narrow angle diagram that permits to discover several dynamic and structure peculiarities of global plasma oscillations in $\mathrm{m} / \mathrm{n}=1 / 1$ mode; ECE equipment is used also for measurement of emission in frequency range of magnetized Langmuir oscillations. Some results of measurements are shown: an unevenness of disturbances motion in space; appearance of rational magnetic surface on half of $\mathrm{q}=1$ surface radius; strong oscillations of longitudinal electron velocity at the central plasma area; stability of distribution function of high energy electrons at plasma edge; spectrum of plasma oscillations in sawtooth regime.
\end{abstract}

\section{Introduction}

ECE diagnostics are used in practice on all tokamaks and stellarators and projects for tokamak reactor ITER. As rule its application is limited by measurement of electron temperature. Goal of this work is to demonstrate many additional possibilities of method for investigation of kinetic properties of thermonuclear plasma. ECE complex on T-10 possesses the set of features which distinguish it from the diagnostics on others installations. First, measurements fulfills simultaneously on $1^{\text {st }}$ and $2^{\text {nd }}$ harmonics. Second, the frequency ranges of every harmonics are essentially broadened down that makes possible to analyse ECE of both main part of electrons (in high optical density conditions) and high energy electrons (in low optical density). Third, ECE measurements perform at the same time in two polarizations (ordinary for $1^{\text {st }}$ resonance and extraordinary for $2^{\text {nd }}$ resonance) that, in definite conditions, enables to determine the total, longitudinal and perpendicular velocity of both main body electrons and electrons of the tail part of distribution. Equipment is used also for registration of magnetized Langmuir oscillations. Some examples of various applications of the ECE apparatus are presented.

\section{Structure and main technical characteristics}

Technically the complex consists of two groups of receivers. First group is the multichannel receivers in the cassette design with double frequency conversion and external local oscillator. Two devices with the manual control have correspondingly $10(1-19$ ГГц) and $12(2-24$ ГГц) channels with $2 \mathrm{GHz}$ interval on odd and even frequencies. New 12 channel receiver with computer control gives possibility to change slightly the secondary carrier frequencies from gyrotron frequencies when the last deviate from the nominal values and also when difference frequencies between gyrotron frequencies 129 and $140 \mathrm{GHz}$ and first heterodyne one intersect with IF bands of different channels. The $1 \mathrm{GHz}$ interval between channels in combination with other receivers provides twice as better space distribution in the profile measurements. Complete set of replacement converters on IMPATT 
or Gann diodes with wide band amplifiers $(25 \mathrm{GHz})$ enables an operation in different ranges of the frequency band $37-170 \mathrm{GHz}$. Low frequency IF band is disposed of by LF filter in converters input. Rejector filters and attenuators on pin-diodes in converters and multichannel receivers input are applied for protection from overload. In some experiments two channel receivers on Michelson interferometer are applied. For all receiver the variable IF band is $200-600 \mathrm{MHz}$. Output band is no less then $0.5 \mathrm{MHz}$. Periodicity of digitization of all signals is $1 \mathrm{mcs}$.

Second group of four receivers with single frequency conversion uses packaged BWO in the capacity of local oscillator. Programmatically controllable high voltage power supply provides change of frequency by given low. The frequency-voltage equivalence is determined by preliminary calibration. Two regime of frequency modulation: sawtooth and stepped - are possible.

First mode (linear scanning) enables to carry out frequency scanning in full range 37 - 178 $\mathrm{GHz}$ during $0.5 \mathrm{~ms}$. The realized amount of frequency points in total spectrum is $32 \mathrm{x} 4=128$. Second mode (stepped mode) is intended to avoid an influence of gyrotron frequencies to the system operation. In such case the coincidence of gyrotron and LO frequency is realized on the step front. The short time $(\sim 20 \mathrm{mcs})$ proximity of gyrotron and LOC frequencies excludes an overload of IF amplifier. The narrow spectral strips are removed when in use the automatic processing. Full spectrum can result for $0.5-2 \mathrm{~ms}$ subject to the amount of selected frequencies and width of spectral band. Receiver IF band is $\pm(50-300) \mathrm{MHz}$. Every receiver is possible to operate on one selected frequency with resolution 1 mcs.

The relative calibration of receivers together with antenna system fulfills by two Ohmic discharges with small difference of magnetic field. The values of field is selected thereby that the frequencies in channels correspond to that of main plasma emission on $1^{\text {st }}$ and $2^{\text {nd }}$ resonances. Signals on quasi-stationary stage are averaged during $\sim 100 \mathrm{~ms}$. Automatic algorithm of linear approximation is used for calculation of sensitivity coefficients. The normalization of sensitivity is put into effect by the spectral SXR measurements along central chord. Fig. 1 illustrates the arrangement of ECE equipment and gyrotron launches on installation.

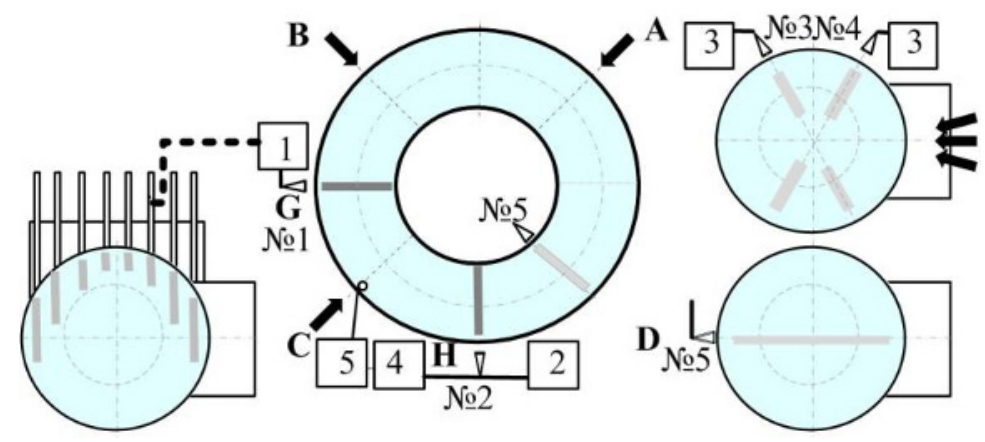

Fig. 1. Arrangement of ECE equipment and gyrotron launches (arrows) on installation. Initial letter identify the T-10 ports. Permanent ECE measurements are make by antennae №1 (G) and 2 (H). Antennae №3 and 4 (A) are for analysis of plasma disturbances motion. ECE measurements from HFS fulfilled by antennae №5 (D).

Interferometer antenna system (C) is used for ECE measurements along lines of equal magnetic field. 1 multichannel receivers, 2 - scanning receivers, 3 - two- and 4 - one cannel receiver, 5 - measurement of plasma noises.

Two similar antennae №1 and 2 are applied for regular measurement of ECE spectra. Both antennae are the beginning of oversize waveguides $\varnothing 20 \mathrm{~mm}$. Antennae are situated in equatorial place at LFS and oriented perpendicularly to the magnetic axis of installation. The beam dividers on polarizing grids direct energy in two pairs of tracts with ordinary and extraordinary polarization. After beam divider, the antenna pass consists of a combination of beam waveguides $\varnothing 20 \mathrm{~mm}$ (with Gaussian mode) and different oversize rectangular waveguides (with a high order waveguide mode). The optical axis of antenna system is adjusted by a laser. The antenna pass №1 possesses the more 
narrow angle diagram $\pm\left(1^{0}-1.5^{0}\right)$ on the half-width than the antenna pass №2 $\pm\left(2-2.5^{0}\right)$. This effect is provided by a more long quasi-optical length and correspondingly more intense diagram sharpening by losses of Gaussian distribution wings.

The antennae №3 и 4 angularly $60^{\circ}$ and $120^{\circ}$ to the equatorial plane are used for an analysis of the disturbance motion in the poloidal and toroidal directions. The antenna №5 is applied for ECE measurement from HFS. The interferometer antenna system is used for ECE measurements along lines with constant magnetic field. The multichannel receiver units without external converter can be used for measurement of the high frequency plasma oscillations in the range $1-24 \mathrm{GHz}$. Electromagnetic loops (an effective size $1-2 \mathrm{~cm}^{2}$ ) insulated from a direct contact with plasma by the ceramics screen are applied for a wave receiving. Later some examples of manifold application of complex will present.

\section{Special applications of ECE diagnostics}

\subsection{Narrow angle antenna diagram}

As it turned out an insufficient narrow antenna diagram distorts the phase relations and space features of the global plasma oscillations [1]. Fig. 2 shows the correct dynamics of ECE signals from LFS and HFS which were obtained by antenna №1 with narrow diagram. Here on-axis ECH is used for oscillations $\mathrm{m} / \mathrm{n}=1 / 1$ intensification. It is obvious that motion of the disturbances around torus is nonuniform (the phase shift is $\sim 120^{\circ}$ ) whereas antenna №2 shows this motion as uniform (phase shift is $\left.\sim 180^{\circ}\right)$. This phenomenon becomes clear by the linear Doppler-effect when the longitudinal electron velocity varies strong in acts of the global plasma oscillations [2].
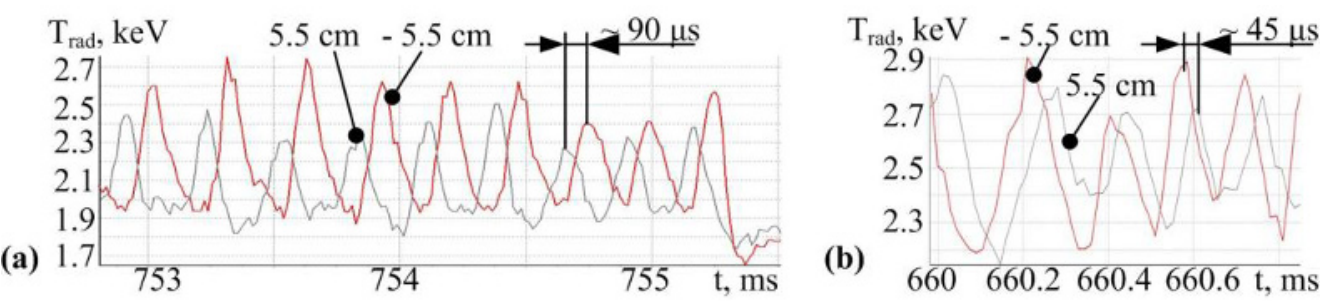

Pис. 2. \#36053. Phase relations of ECE signal oscillations on LFS and HFS (antenna №1).

Periods of ECE and SXR amplitude oscillations can change during a discharge more than two times $(\sim 330-160 \mathrm{mcs}, \sim 3-6 \mathrm{kHz})$. But then the transition time from LFS to HFS (fig. 2a) or from HFS to LFS (2b) can changes no more than $10-15 \%$. The frequencies of spiral motion calculated by the velocities of their poloidal motion at the central plasma area coincide confidently with the current oscillation frequencies measuring by the magnetic probes at the plasma periphery. The spiral motion of disturbances $\mathrm{m} / \mathrm{n}=1 / 1$ happen with the eigen frequencies of the current oscillations $\mathrm{f}_{1 / 1}^{\mathrm{l}} \sim$ $6.3, \mathrm{f}_{1 / 1}^{2} \sim 12$ и $^{4}{ }_{1 / 1} \sim 23 \mathrm{kHz}$. Top index gives the harmonic number, bottom - the mode structure. Those frequencies appear with the beginning of the sawtooth process and preserve with small deviation all discharge time. It was discovered in regime with excitation of several global modes that a relation of their first adjacent in space harmonics is the prime number: $\mathrm{f}_{1 / 1}^{1} / \mathrm{f}_{3 / 2}^{1}=\mathrm{f}_{3 / 2}^{1} / \mathrm{f}_{2 / 1}^{2}=\mathrm{f}_{2 / 1}^{1}$ $/ \mathrm{f}_{3 / 1}^{1}=3 / 2$. The eigen frequencies do not depend on electron temperature, density and $\mathrm{ECH}$ power and remain during all discharge [2].

Profile measurements show that global plasma oscillations possess by certain thin space structure. It is good known that rational magnetic surface $\mathrm{q}=1(\mathrm{r} \sim 8.5 \mathrm{~cm})$ is accompanied by sharp jumps of the temperature gradient (on HFS) and by the flattened zone on LFS. High space resolution with on-axis ECH makes possible to discover similar effects on the half of $\mathrm{q}=1$ radius $\mathrm{r} \sim 4.5 \mathrm{~cm}$ (fig. $3 a)$ that shows to appearance a new rational surface $\mathrm{q}=1$ * when oscillations are being strong. Space 
coordinate where global oscillations change their phase coincide with the positions of rational surfaces. Global oscillations with frequency $\mathrm{f}_{\text {mod }}^{1} \sim 3.6 \mathrm{kHz}$ have similar phases at all area inside surface $\mathrm{q}=3 / 2$ on LFS and at zone between $\mathrm{q}=3 / 2$ and new peculiarity $\mathrm{r} \sim-4.5 \mathrm{~cm}$ on HFS (fig. $3 b$ ). Inside this surface, tree cycles of oscillations are observed during period of $\mathrm{f}^{1}$ mod that corresponds to second eigen frequency $\mathrm{f}_{1 / 1}^{2} \sim 12 \mathrm{kHz}$ which is being the driving frequency under on-axis $\mathrm{ECH}$. It means that new rational surface $\mathrm{q}=1^{*}$ is second structural and frequency harmonic of the master surface $q=1$ which is responsible for generation of first oscillation harmonic $\mathrm{f}_{1 / 1}^{1} \sim 6.3 \mathrm{kHz}$ when global oscillations have moderate amplitudes $[2,3]$. It should emphasize that positions of rational surfaces remain invariable for a while global oscillations exist. Relations of the nearest rational surfaces positions as for eigen frequencies is prime number: $\mathrm{r}_{\mathrm{q}=3} / \mathrm{r}_{\mathrm{q}=2}=\mathrm{r}_{\mathrm{q}=2} / \mathrm{r}_{\mathrm{q}=3 / 2} / \mathrm{r}_{\mathrm{q}=3 / 2} / \mathrm{r}_{\mathrm{q}=1}=3 / 2$.
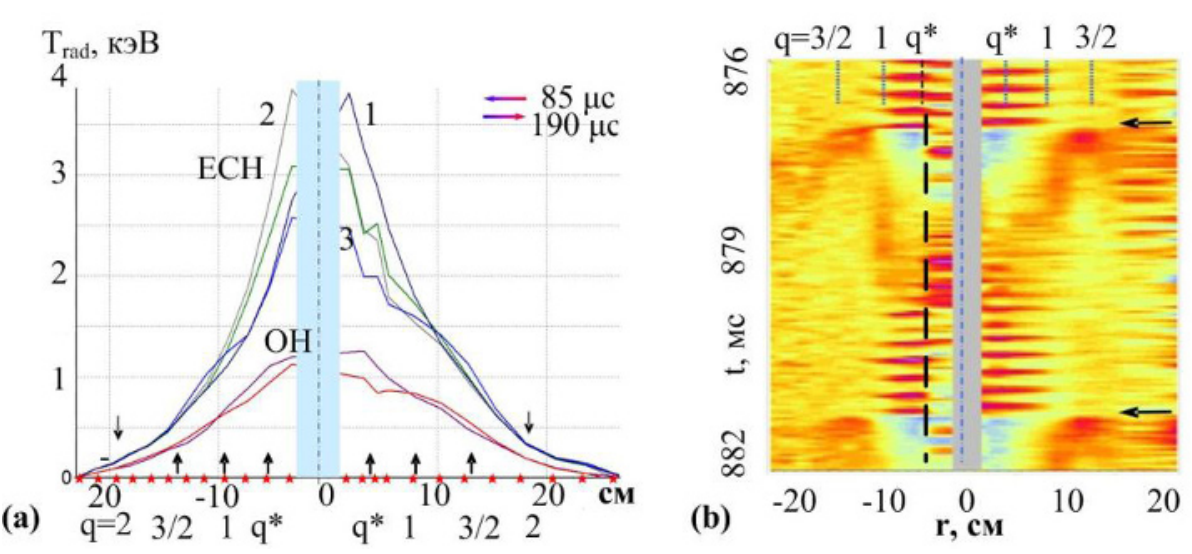

Fig. 3. $\# 36055$. Space structure of $m / n=1 / 1$ oscillations. (a) - Electron temperature profile dynamics. Lower curves - Ohmic stage, higher curves - with ECH: 1 - maximum on LFS, 2 - maximum on HFS, 3 - just after disruption, remainder curves - intermediate states. Vertical arrows show positions of rational magnetic surfaces. Horizontal arrows show character time of disturbances motion from LFS to HFS and on the contrary. Stars represent space positions of registration zones by different channels. Colored zone nearby the plasma center covers area where measurements are impossible by gyrotron frequencies. (b) - phase peculiarities illustrations of temperature oscillations inside and outer coordinate $4.5 \mathrm{~cm}$ on HFS (dotted line). Horizontal arrows show time of internal disruptions.

\subsection{ECE in O- and X-polarization from plasma with high optical density}

In order to verify the supposition that electron distribution function widens and compress along longitudinal velocity we used the simultaneous ECE measurements in ordinary ( $1^{\text {st }}$ resonance) and extraordinary $\left(2^{\text {nd }}\right.$ resonance) polarization from plasma with high optical density $\tau \sim 10$ where emission is provided by main part of distribution. In this case ECE in X-mode is created only by perpendicular electron velocity and ECE in O-mode - by both perpendicular and longitudinal velocity. Simultaneous ECE measurements on $1^{\text {st }}$ and $2^{\text {nd }}$ harmonics are fulfilled on multiple frequencies with using of common antenna and recorder (from common points in space) [1, 4]. Under low level of global oscillations $\left(\mathrm{P}_{\mathrm{ECH}} \sim 0.2-0.25 \mathrm{~kW}\right)$, the change of amplitude is practically the same as it must be in case of the thermodynamically equilibrium plasma that corresponds to the usually applicable linear ECE theory. However oscillations in X- and O-mode have different phases. On LFS oscillations of signals in O-mode outstrip signals of X-mode on 40 - 45 mcs and drop earlier before a disruption. Those regular effects show to periodical variation of distribution function anisotropy in the acts of global oscillations. In disturbance motion around torus, the longitudinal electron velocity relaxes on LFS so distribution function on HFS proves "compressed" in the longitudinal direction.

A difference in signal dynamics are being considerably stronger under high ECH power $(0.8$ $-1.2 \mathrm{MW}$ ) (fig. 4). The growth rate of O-ECE after disruption is $3-5$ times more than X-ECE and 
after decreases. Maximal amplitudes of oscillations in O-mode can exceed $100 \%$ relatively to the background. Particularly big difference in X- and O-mode dynamics can be observed during $40-80$ ms after ECH start (fig. 4). Dashed lines lower two upper curves show to an equality of the background levels of O- and X-ECE just after internal disruptions. It means that distribution function in this time is in state of the thermodynamic equilibrium. The same curve lower HF signal which represents the total power of plasma noises shows that their dynamics in first $\mathrm{ECH}$ stage is similar to that of O-ECE. It corresponds to that that maximal longitudinal deformation of distribution function happens just in first ECH stage. The evaluation shows that oscillations of the longitudinal velocity in main part of distribution can be comparable with its average value during "saw" period. This phenomenon shows to kinetic nature of global oscillations and internal disruptions.
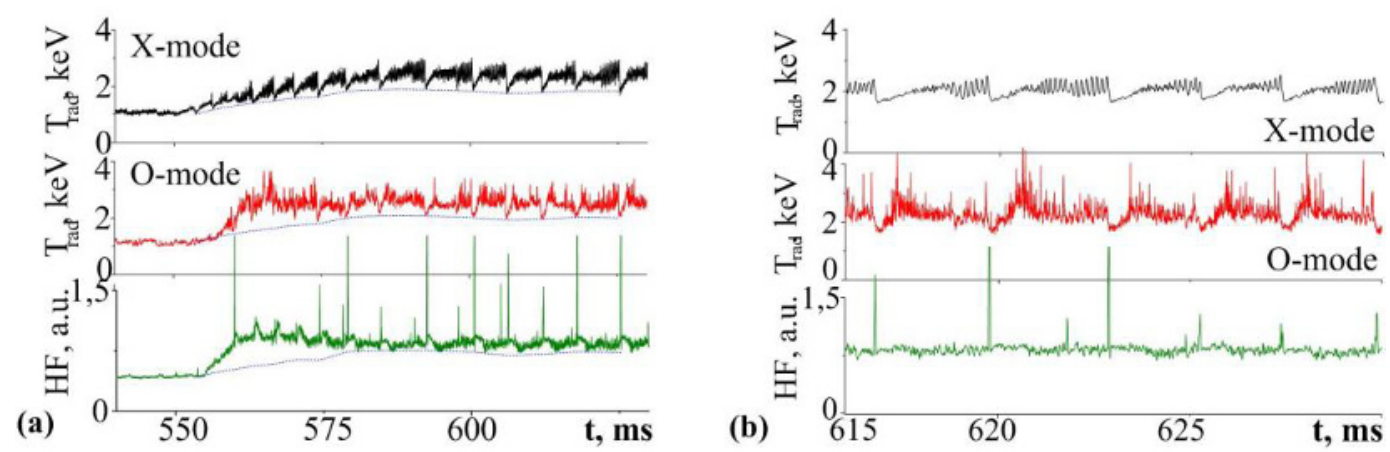

Рис. 4. \#44631. Signals O-ECE, X-ECE and plasma noises HF (0.5 - $16 \mathrm{GHz})$ in regime with on-axis ECH. (a) - first stage of heating, (b) - quasi-stationary stage.

\subsection{ECE of high energy electrons}

General view of spectra on $1^{\text {st }}$ and $2^{\text {nd }}$ resonances is shown at fig. 5 in $3 D$-format. Besides the thermal emission of main plasma in the frequency ranges higher than cyclotron frequency on the outer boundary of plasma ( $58 \mathrm{GHz}$ for $1^{\text {st }}$ harmonic and $116 \mathrm{GHz}$ for $2^{\text {nd }}$ ), the measured spectra content also components in the low frequency range up to $38 \mathrm{GHz}$ for $1^{\text {st }}$ harmonic and $78 \mathrm{GHz}$ for $2^{\text {nd }}$ harmonic $[5,6]$. It should determine the nature of this emission. It can be cyclotron emission of the high energy electrons shifted down by relativistic and linear Doppler-effect or electromagnetic emission which is result of potential plasma wave transformation.
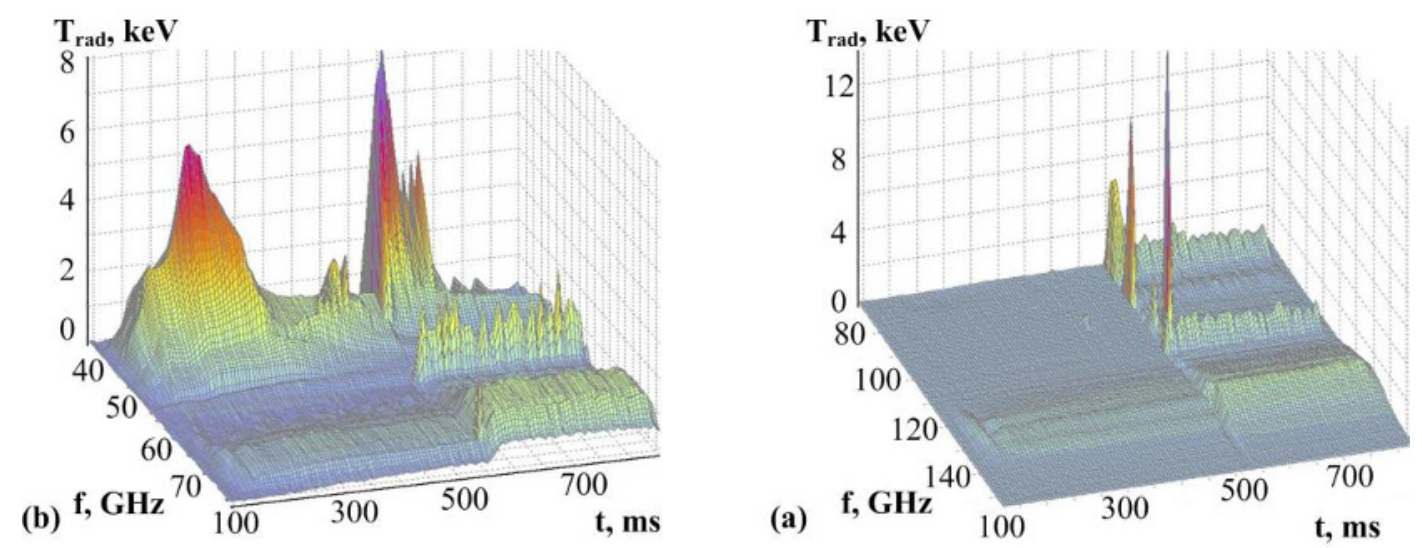

Fig. 5. \#36055. 3D-view of full spectra. (a) $-1^{\text {st }}$ resonance, (b) $-2^{\text {nd }}$ resonance. 
Two branches of magnetized plasma oscillations are known: high frequency (electron Bernstein branch) and low frequency (Triwelpiece-Gould mode). First in our conditions has range of the eigen frequencies $\omega_{\mathrm{ce}} \leq \omega_{\mathrm{k}}^{(1)} \leq\left(\omega_{\mathrm{pe}}^{2}+\omega_{\mathrm{ce}}^{2}\right)^{1 / 2}$ where $\omega_{\mathrm{ce}}-$ electron cyclotron frequency, $\omega_{\mathrm{pe}}-$ Langmuir electron frequency. Right term of the inequality is known as upper hybrid frequency. This branch cannot give deposit to emission on the frequencies lower than $57 \mathrm{GHz}$. Second branch has the frequency range $\omega_{\text {low }} \leq \omega_{\mathrm{k}}^{(2)} \leq \omega_{\mathrm{pe}}$. Low frequency boundary is closed to Langmuir ion frequency $\omega_{\mathrm{pi}}$. We can realize the conditions by a reduction of plasma density when upper frequency of second branch in all plasma volume is lower than the lowest frequency in measured spectrum. The results of experiment are present on fig. 6 . The graph (a) illustrates a change of density profile with a reduction of average value. The graphs (b) and (c) show almost exponential growth of signals on the quasistationary stage that corresponds to the good known phenomenon - an increase of the high energy electron amount under a decrease of plasma density. The increase of signal (4) up to the end of discharge illustrates a transition of plasma to so called runaway regime.

The upper boundary of second branch is $40 \mathrm{GHz}$ at the plasma center and $22 \mathrm{GHz}$ at the plasma edge for the conditions corresponding to the curves (2). The values for the conditions (3) and (4) are less than $29 \mathrm{GHz}$ at the plasma center and $10 \mathrm{GHz}$ at the plasma edge. It should note that the real measuring on T-10 the emission of second branch is in the interval $0.5-15 \mathrm{GHz}$ [7]. Therefore the investigated spectrum into low frequency range $38-58 \mathrm{GHz}$ is really O-mode of first ECE harmonic of high energy electrons.

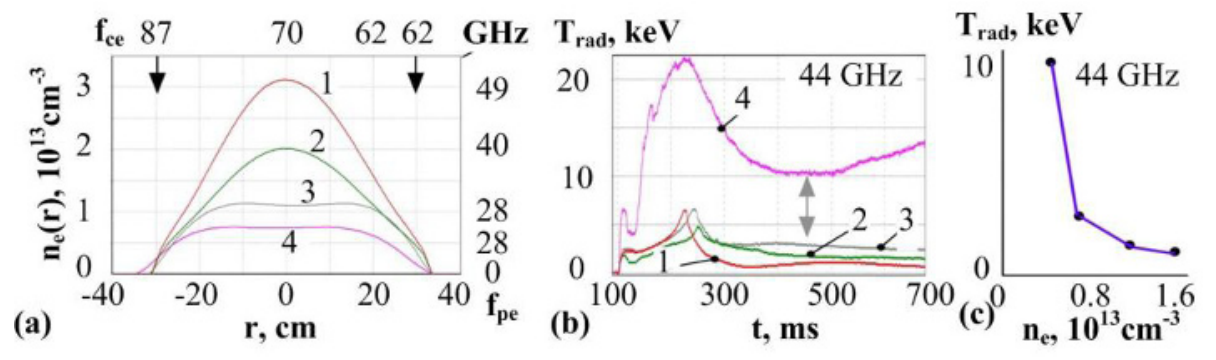

Pис. 6. \#59838 - 59841. (a) - Change of density profile for successive reduction of its average value $n_{\mathrm{e}}: 1$ $1.6,2-1.0,3-0.7,4-0.5 \cdot 10^{13} \mathrm{~cm}^{-3}$. Vertical arrows mark the boundary of current channel. (б) Signals on frequency of maximum in spectrum $\sim 44$ ГГц. (в) - Dependence of signal amplitudes on average plasma density in quasi-stationary stage. Graphs (а) and (в) obtained in time shown on fig. 6 b by double-sided arrow.

All peculiarity of spectrum in frequency range $78-116 \mathrm{GHz}$ remain under reduction of magnetic field to $23 \mathrm{kGs}$ but in this case denoted spectrum is higher than the minimal value of upper hybrid frequency which is top boundary of electron Bernstein branch. Thereby signals in range of doubled frequency near to thermal part of spectrum of second harmonic are ECE of high energy electrons.

In order to extract distribution function of high energy electron population we must determine their location in space. Fig. 7 presents the dynamic measurements of ECE spectrum and density profile at the discharge with monotonous growth of average plasma density. On-axis ECH starts on $550 \mathrm{~ms}$. It can see at fig. 7a that the low frequency part of spectrum cuts off gradually owing to the broadening of the inaccessibility area for $\mathrm{O}$-waves. Under more low but invariable density amplitudes of signals decrease to certain stationary level but form of spectrum remains up to the end of discharge. Comparison ECE spectra and density profile dynamics shows that the emitted layer with a depth no more than $2-3 \mathrm{~cm}$ is on LFS of column inside the current channel and coincides with the position of surface $q=3$. ECE with frequencies $39-60 \mathrm{GHz}$ propagates free between the plasma cut-off and chamber wall. Per contra ECE with frequencies higher $60 \mathrm{GHz}$ is inaccessible for observation by absorption of main plasma. 
The extraction of distribution function by the measured spectra [8] shows that emitting electrons possess the average total energy $\sim 150-200 \mathrm{keV}$ with relation of transverse and longitudinal energy $\mathrm{W}_{\perp} / \mathrm{W}_{/ /} \sim 10$. They are trapped particles. A consistency of their spectrum during discharge, independence of it on electron temperature and density, but the linearly dependence of the spectral width on the value of magnetic field show to that this high energy electron component results by a transport of potential plasma waves from the central plasma area [9]. Experimental data correspond to the theoretical solution on the stationary electron distribution function which forms by the plasma waves [10].


Puc. 7. (a) - Change of $1^{\text {st }}$ harmonic ECE spectrum in regime with growing plasma density. Triangle marks position of surface $\mathrm{q}=3$. (b) - variation of density profile. Rectangles show size of ring limiter. Vertical lines restrict the diameter of current channel by rail limiter

\subsection{Measurements of plasma noises}

ECE receivers without external converters used also for measurements of emission in range of magnetized Langmuir oscillations 1-24 GHz. Small loop electromagnetic antennae insulated by ceramics from direct contact with plasma are applied. Fig, 8 presents dynamics of plasma noises during discharge. Breakdown stage where amplitudes of signals more than order of value higher than at this picture is excluded (fig. 8a).
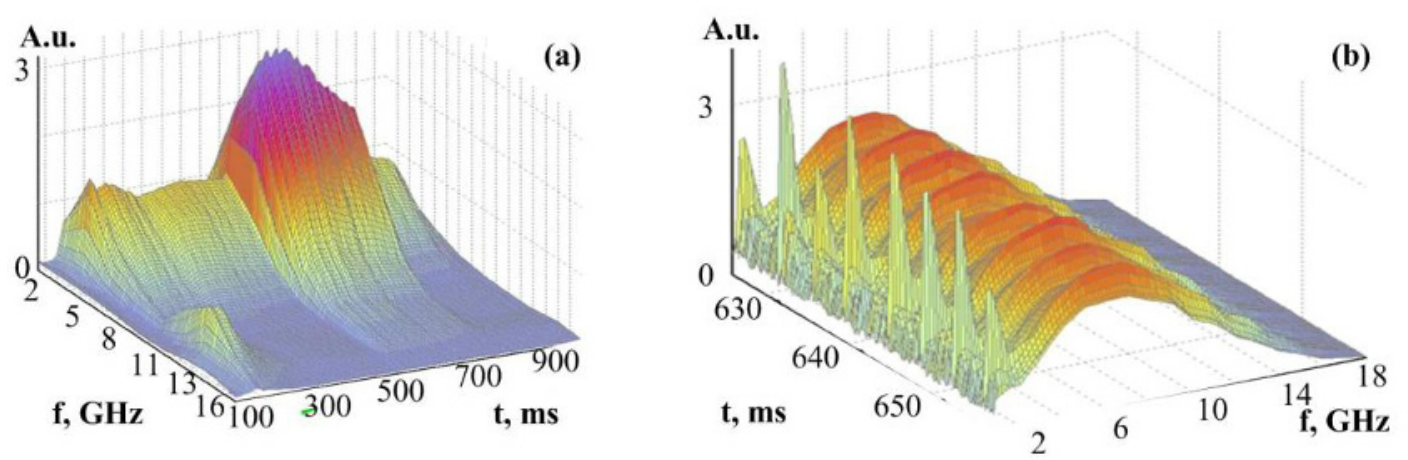

Fig. 8. Total spectrum of plasma oscillations. (a) - Change of spectrum during discharge. (b) - Spectrum dynamics under sawtooth process.

In stationary stage, plasma noise level can exceed maximal level of thermal ECE. Stable spectra has the bell like form. In Ohimic regime, maximum of spectrum is near to $4-5 \mathrm{GHz}$. Under 
$\mathrm{ECH}$, signals grow aproximately proportional to electron temperature. Maximum shifts to $6-8$ GHz. The low frequency part of spectrum changes during every cycles of sawtooth process. Internal disruptions are accompanied by the strong splashes on the low frequency boundary of spectrum that testifies on the kinetic nature of instability. They surpass an intensity of plasma noise background in one-two orders of value [11]. We use the property of spectrum to grow strong on the low frequency boundary just in disruption. If spectrum corresponds to the magnetized Langmuir oscillations this boundary is $\omega_{\text {low }}^{(2)}=\omega_{\mathrm{pi}} /\left(1+\omega_{\mathrm{pe}}^{2} / \omega_{\mathrm{ce}}^{2}\right)^{1 / 2} \sim \omega_{\mathrm{pi}}[12]$. So far as $\omega_{\mathrm{pi}}=\omega_{\mathrm{pe}}\left(\mathrm{m}_{\mathrm{e}} / \mathrm{m}_{\mathrm{i}}\right)^{1 / 2}$ we can find space position of layer where electron density is equal to corresponding value.



Fig. 9. Experimental identification of space and frequency boundary conditions for plasma waves. 1 - Ohmic discharge, 2 - with ECH.

Fig. 9 illustrates a decrease of density at the central plasma area under powerful ECH. Vertical dashed lines show the position of surface $q=1$ by ECE and SXR measurements of temperature inversion in the internal disruption. The arrows mark the measured low frequensy boundaries of spectra for different time. Both value show to the same radial coordinate which coinsides with $q=1$ position. We concede some possible errors in local density values and $\mathrm{q}=1$ position (the horizontal and vertical dashed lines) but the discovered correlation remains under a considerable increase of average density. Thereby the electron density in the vicinity of rational surface $\mathrm{q}=1$ forms the eigen frequency spectrum of Langmuir waves.

This work was supported by the Russian Ministry of Education and Science (Contract No. 16.518.11.7004) and Rosatom (Contract No. H.4f.45.90.11.1021).

1. V.I. Poznyak, T.V. Gridina, V.V. Piterskii et al., Proc. of $16^{\text {th }}$ Joint Workshop on ECE and ECH, Sania, China, p. 114 (2010)

2. V.I. Poznyak, T.V. Gridina, V.V. Piterskii at al., Plasma Physics Report, v. 8, (2012) to be published

3. V.I. Poznyak, A.M. Kakurin, T.V. Gridina at al., Plasma Physics Report, v. 9 (2012) to be published

4. V.I. Poznyak, V.V. Piterskii, G.N. Ploskirev at al., Proc. of $15^{\text {th }}$ Joint Workshop on ECE and ECH, Yosemite National Park, California, USA, p. 136 (2008)

5. V.I. Poznyak, V.V. Piterskii, G.N. Ploskirev at al., Proc. of $15^{\text {th }}$ Joint Workshop on ECE and ECH, Yosemite National Park, California, USA, p. 256 (2008)

6. V.I. Poznyak, T.V. Gridina, V.V. Pitersky at al., Problems of Atomic Science and Engineering, Ser. Thermonuclear Fusion (in Russian), v. 4, p. 90 (2011)

7. V.I. Poznyak, A.A. Bagdasarov, V.V. Piterskii and A.N. Yakovets, Proc. of $10^{\text {th }}$ Joint Workshop on ECE and ECH, Ameland, Netherlands, p. 155 (1997)

8. P.V. Minashin, A.B. Kukushkin, V.I. Poanyak, Proc. of $17^{\text {th }}$ Joint Workshop on ECE and ECH, Deurne, Netherlands (2012)

9. Poznyak V.I., Gridina T.V., Piterskii V.V. at al., Proc. of $17^{\text {th }}$ Joint Workshop on ECE and ECH, Deurne, Netherlands (2012)

10. L. Mushcietti, K. Appert, J. Vaclavic, Phys. Fluids, v. 25, p. 1187 (1982)

11. V.I. Poznyak, A.A. Bagdasarov, V.V. Piterskii et al., Proc. of $15^{\text {th }}$ Int. Conf. on Plasma Phys. and Control. Nuclear Fusion Research, Seville, Spain (1994), Nuclear Fusion, v. 2, p. 169 (1995)

12. A.I. Akhiezer, I.A. Akhiezer, R.V. Polovin at al. // Plasma Electrodynamics, Ed. by A.I. Akhiezer, Oxford, New York, Pergamon Press (1975) 